Pneumologe 2007 · 4:233-240

DOI 10.1007/s10405-007-0155-y

Online publiziert: 26. Mai 2007

(c) Springer Medizin Verlag 2007

Redaktion

D. Reinhardt, München
P. Latzin · G. Hafen

Abteilung für pädiatrische Pneumologie, Inselspital,

Universitätskinderklinik Bern, Schweiz

\begin{abstract}
Das respiratorische System erfährt während der Säuglingszeit dramatische Veränderungen. Prä- und postnatale Ereignisse beeinflussen Wachstum und Entwicklung der Atemwege und des Lungenparenchyms [6]. Eine eingeschränkte Lungenfunktion bei Neugeborenen führt zu anhaltender Funktionseinschränkung und gehäufter respiratorischer Morbidität im späteren Leben (sog.,/Tracking"; $[5,7])$. Im Folgenden werden die gängigen Methoden der Lungenfunktionsmessung beim Säugling und Kleinkind dargestellt, und es wird auf die Besonderheiten der Lungenfunktionsmessung in diesem Alter eingegangen.
\end{abstract}

\section{Anspruch an Lungenfunktion im Säuglingsalter}

Die Unterschiede der Lungenfunktionsmessung im Säuglingsalter im Vergleich zum Erwachsenen und Schulkind liegen v. a. darin, dass der Patient nicht kooperationsfähig ist und dass deutlich kleinere Volumina bewegt werden, die eine extreme

\section{Abkürzungen}

\begin{tabular}{|ll}
\hline CF & zystische Fibrose \\
chronische Lungenerkrankung & $\begin{array}{l}\text { des Frühgeborenen } \\
\text { FOT }\end{array}$ \\
\hline "forced oscillation technique" & funktionelle Residualkapazität \\
\hline MBW & "multiple-breath-washout" \\
\hline MOT & "multiple occlusion technique" \\
\hline NO & Stickstoffmonoxid \\
\hline NREM & "non-rapid-eye-movement" \\
\hline RTC & "rapid thoracic compression" \\
\hline SOT & "single occlusion technique" \\
\hline
\end{tabular}

\title{
Lungenfunktion im Säuglings- und Kleinkindalter
}

Messgenauigkeit sowie einen minimalen Totraum der Geräte erfordern. Die Fragestellungen im Säuglingsalter sind hingegen vergleichbar mit denen im späteren Leben: - frühe Diagnose von Lungenerkrankungen,

- Überwachung des Krankheitsverlaufs,

- Effekt von therapeutischen Interventionen,

- Wachstum der Lunge bzw. Entwicklung der Erkrankung,

- Identifizierung von Hochrisikokindern.

\section{Messbedingungen}

Kürzlich wurden ERS/ATS-Standards zur Lungenfunktionsmessung bei Säuglingen publiziert, die detailliert auf die Voraussetzungen der Messbedingungen und den Zustand des Kindes eingehen [9, 10]. Die wichtigsten Merkmale sind:

- Durchführung nach standardisierten Messbedingungen zur Gewährleistung der Vergleichbarkeit und Sicherheit des Kindes,

- ruhiger Schlafzustand des Kindes (NREM-Phase),

- exakte tägliche Kalibrierung der Messeinheit für höchste Genauigkeit,

- minimaler technischer Totraum ( $<2 \mathrm{~mL} / \mathrm{kg} \mathrm{KG)}$ und kleinstmöglicher Widerstand der Messeinheit, um normale Atemlage und Atemmuster zu gewährleisten.

\section{Messparameter und entsprechende Methoden}

Durch die Tatsache, dass die Kinder in diesem Alter nicht kooperationsfähig sind, können einige herkömmliche Lungenfunk- tionstechniken, wie z. B. die forcierte spontane Ausatmung im Rahmen einer Spirometrie, im Säuglingsalter nicht angewandt werden. Andere, früher gebräuchliche $\mathrm{Me}-$ thoden, wie z. B. die „Rapid-thoracic-compression-Technik" in Sedation, verlieren in letzter Zeit zunehmend an Bedeutung, da die Akzeptanz dieser Methode bei den Eltern eher gering ist. Die $\bullet$ Tab. 1 gibt eine Übersicht über Parameter der Lungenfunktion, die im Säuglingsalter gemessen werden können, sowie die entsprechenden Messmethoden. Außerdem sind beispielhaft verschiedene Erkrankungen aufgeführt, bei denen die genannten Messparameter von Interesse sind.

\section{Fluss-Volumen-Kurve}

Die am einfachsten durchführbare Messung während des Säuglingsalters ist die Fluss-Volumen-Kurve. Dabei atmet das Kind in Ruhe, und die Fluss-VolumenKurve kann direkt mittels eines Flussaufnehmers (Pneumotachograph oder Ultraschallsensor) und über eine Gesichtsmaske oder indirekt mittels der sog. Induktionsplethysmographie bestimmt werden [3]. Aus der Fluss-Volumen-Kurve können dann analog zu Schulkindern oder Erwachsenen die gängigen Parameter abgeleitet (• Abb. 1) und für die Beurteilung der Fragestellung verwendet werden [3]. Kürzlich konnte z. B. bei 616 Kindern gezeigt werden, dass ein höheres $\mathrm{T}_{\text {PTEF }} /$ $\mathrm{T}_{\mathrm{E}}$ nach Geburt mit häufigerem Auftreten von Asthma bronchiale während der ersten 10 Lebensjahre vergesellschaftet ist [15]. Diese Daten müssen jedoch vorsichtig interpretiert werden, da es sich bei $\mathrm{T}_{\mathrm{PTEF}} / \mathrm{T}_{\mathrm{E}}$ um einen Mischparameter aus Mechanik und Atemkontrolle handelt. 
Tab. 1 Übersicht verschiedener Fragestellungen und Erkrankungsbeispiele mit entsprechenden Messmethoden

\begin{tabular}{|c|c|c|}
\hline Fragestellung & Störung (typische Erkrankung) & Messmethoden \\
\hline Obstruktion? & $\begin{array}{l}\text { Obstruktive Erkrankungen (CLD, } \\
\text { Asthma bronchiale) }\end{array}$ & $\begin{array}{l}\text { Fluss-Volumen-Kurve } \\
\text { (Ruheatmung) } \\
\text { Mechanik (s. unten) }\end{array}$ \\
\hline Lungenvolumen? & $\begin{array}{l}\text { Lungenwachstum (CLD) } \\
\text { Überblähung (Asthma bronchiale) }\end{array}$ & $\begin{array}{l}\text { Plethysmographie } \\
\text { Gasverdünnung }\end{array}$ \\
\hline Gasverteilung? & Verteilungsstörung (CF, CLD) & Gasverdünnung \\
\hline Entzündung der Atemwege? & $\begin{array}{l}\text { Asthma bronchiale } \\
\text { CLD } \\
\text { Zilienfunktionsstörung } \\
\text { Gastroösophagealer Reflux }\end{array}$ & $\begin{array}{l}\text { NO-Messung } \\
\text { Atemkondensat }\end{array}$ \\
\hline Atemregulation? & Unreife der Atemzentren & Fluss-Volumen-Kurve \\
\hline Atemmechanik? & $\begin{array}{l}\text { Entwicklungsstörung der Atemwege } \\
\text { (Hypoplasie, CLD) }\end{array}$ & $\begin{array}{l}\text { Okklusion } \\
\text { Plethysmographie } \\
\text { "Forced oscillation" } \\
\text { Interrupter-Technik }\end{array}$ \\
\hline
\end{tabular}

\section{Tab. 2 Neue, nichtinvasive Messmethoden}

\begin{tabular}{|c|c|c|}
\hline Technik & Messung & Einsatzbereich \\
\hline EIT & $\begin{array}{l}\text { Impedanzänderungen über } \\
\text { der Lunge }\end{array}$ & $\begin{array}{l}\text { Gasverteilung } \\
\text { Unmittelbare Therapiekontrolle } \\
\text { Seitenunterschiede }\end{array}$ \\
\hline EMG & $\begin{array}{l}\text { Aktivität der Atemhilfsmusku- } \\
\text { latur }\end{array}$ & $\begin{array}{l}\text { Unterscheidung von zentralen Störungen oder } \\
\text { Entwicklungsverzögerungen der Atemregulation und } \\
\text { peripheren Störungen der Atemmechanik }\end{array}$ \\
\hline
\end{tabular}

( Die am einfachsten durchführbare Messung während des Säuglingsalters ist die Fluss-Volumen-Kurve

Die Vorteile dieser Methode liegen darin, dass sie einfach, nicht invasiv und ohne Sedation und Kooperation durchgeführt werden kann und dass neben Informationen über die Atemwegsmechanik auch Informationen über die Atemregulation gewonnen werden können. Nachteile und Einschränkungen dieser Methode beinhalten v. a., dass bei Einschränkungen der Fluss-Volumen-Kurve schlecht zwischen Problemen der zentralen Atemregulation und Problemen der peripheren Atemwegsmechanik unterschieden werden kann.

\section{Volumenmessung}

Die Messung des Lungenvolumens ist ein wichtiger Bestandteilteil der Lungenfunktionsprüfung, da sich Resistance, Compliance und exspiratorische Flüsse abhängig vom Lungenvolumen ändern. Dies trifft im Säuglinsalter aufgrund der relativen Thoraxinstabilität stärker zu als im spä- teren Leben. Bei Neugeborenen und Säuglingen ist die funktionelle Residualkapazität (FRC), das Volumen am Ende einer normalen Ausatmung, das einzige zuverlässig messbare Lungenvolumen. Eine korrekt gemessene FRC ist extrem wichtig, um andere Lungenfunktionsergebnisse richtig interpretieren zu können. Die FRC kann mithilfe von 2 verschiedenen Messmethoden bestimmt werden:

- Ganzkörperplethysmographie

$\left(\mathrm{FRC}_{\mathrm{box}}\right)$ misst das gesamte im Thorax enthaltene Gasvolumen inklusive der sog. „Trapped-gas-Bezirke“ [29],

- im Gegensatz zu Techniken der Gasverdünnung $\left(\mathrm{FRC}_{\mathrm{MBW}}\right)$, die nur das mit den Atemwegen kommunizierende Gasvolumen messen [24].

Die Anwendung von beiden Methoden erlaubt so eine Bestimmung des „trapped gases".

Die physikalischen Prinzipien der Messung der FRC $_{\text {box }}$ basieren wie bei der Erwachsenenbodyplethysmographie auf dem Boyle-Mariotte-Gesetz. Während 2-3 Atemmanövern wird eine Okklusion, beginnend am Ende einer Inspirati- on, durchgeführt. Die resultierenden Änderungen im Thoraxvolumen können direkt über einen Flussaufnehmer (volumenvariabler Plethysmograph) oder indirekt als Druckänderungen in dem volumenkonstanten Plethysmographen gemessen werden. Aus den resultierenden Kurven kann dann FRC $_{\text {box }}$ berechnet werden. Obwohl diese Methode es erlaubt, das $\mathrm{FRC}_{\text {box }}$ rasch und reproduzierbar $\mathrm{zu}$ messen, benötigt die Anwendung komplizierte Geräte und in der Durchführung erfahrene Untersucher [19]. Deshalb ist sie bei kranken Säuglingen, insbesondere im intensivmedizinischen Bereich, kaum einsetzbar [20].

Die Techniken der Gasverdünnung haben den Vorteil, dass sie prinzipiell auch beim beatmeten Säugling durchgeführt werden können. Die „Multiple-breathwashout-Methode" (FRC MBW $)$ misst in einem geschlossenen System mittels eines Indikatorgases (inertes Gas, z. B. Helium, $\mathrm{SF}_{6}$ ) den Konzentrationsausgleich zwischen dem unbekannten Lungenvolumen und einem bekannten Volumen. Das Gas kann entweder direkt z. B. mittels eines Massenspektrometers gemessen werden [2] oder indirekt z. B. mittels Infrarotsensor oder Ultraschallkopf [27]. Für letztere Methode liegt ein im Säuglingsalter zugelassenes kommerziell erwerbbares System vor (• Abb. 2; [4]). Die Hauptprobleme dieser Methode sind die Anforderungen an die Geräte. Der Totraum sollte bei diesen Messungen unbedingt minimal gehalten und in die Interpretation der Ergebnisse einbezogen werden. Die Stickstoff-Gasverdünnungsmethode mit Einatmung eines stickstofffreien Gases (z. B. reiner Sauerstoff; [24]) wird im Säuglingsalter zunehmend seltener angewandt, da reiner Sauerstoff über die Stimulation von peripheren Chemorezeptoren zu einer Verminderung des Atemantriebes und Abnahme der Atemzugsvolumina mit erniedrigter FRC führen kann [20].

\section{Gasverteilung}

Bei der Gasverdünnungsmethode erhält man zusätzlich zur FRC auch Daten über die Effizienz des Gas-Mixing in der Lunge. Damit können Aussagen über die Inhomogenität der Gasverteilung getroffen werden [25]. Der momentan gebräuch- 
Hier steht eine Anzeige.

黛 Springer 


\section{Leitthema}

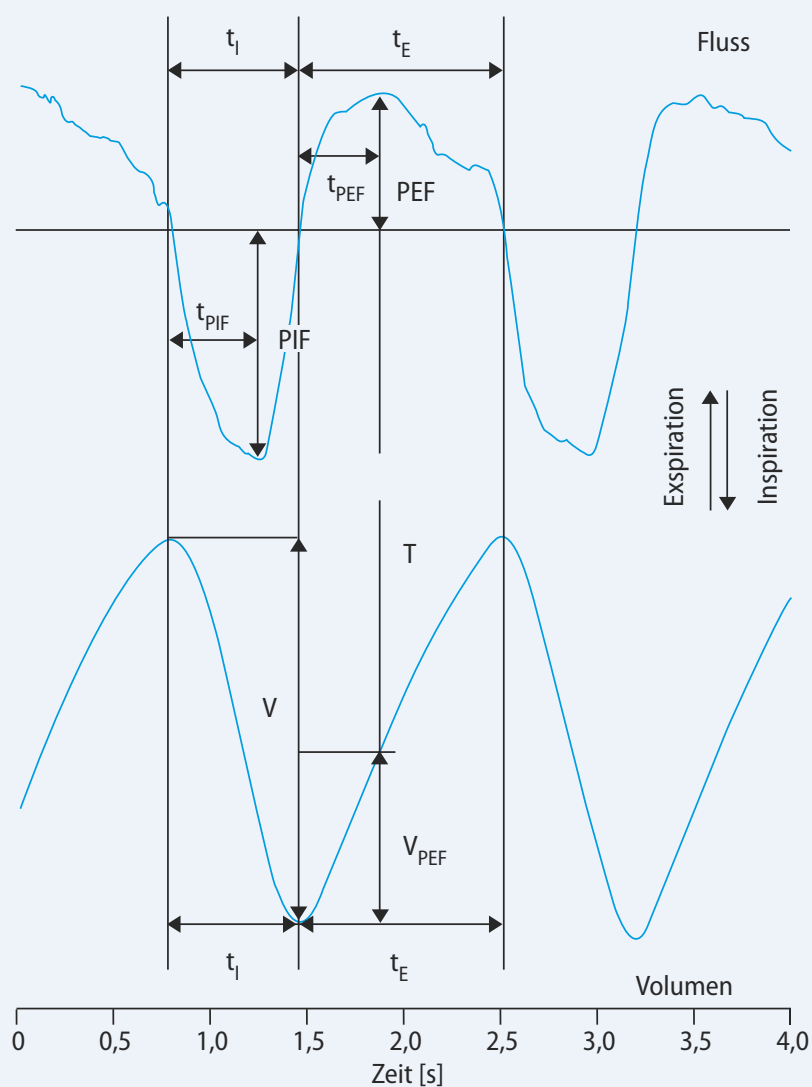

Abb. $1 \Delta$ Parameter eines Atemflusssignales eines 6 Monate alten Säuglings. Die Dauer eines Atemzyklus setzt sich aus der Inspirationszeit $\left(t_{1}\right)$ und der Exspirationszeit $\left(\mathrm{t}_{\mathrm{E}}\right.$ ) zusammen. (PIF maximaler Atemzugsinspirationsfluss, PEF maximaler Atemzugsexspirationsfluss, $t_{\mathrm{PIF}}$ Zeit vom Inspirationsbeginn bis zum PIF, $t_{\mathrm{PEF}}$ Zeit vom Exspirationsbeginn bis zum PEF, $V_{\top}$ Atemzugvolumen, $V_{\mathrm{PEF}}$ am PEF exspiriertes Volumen [26])

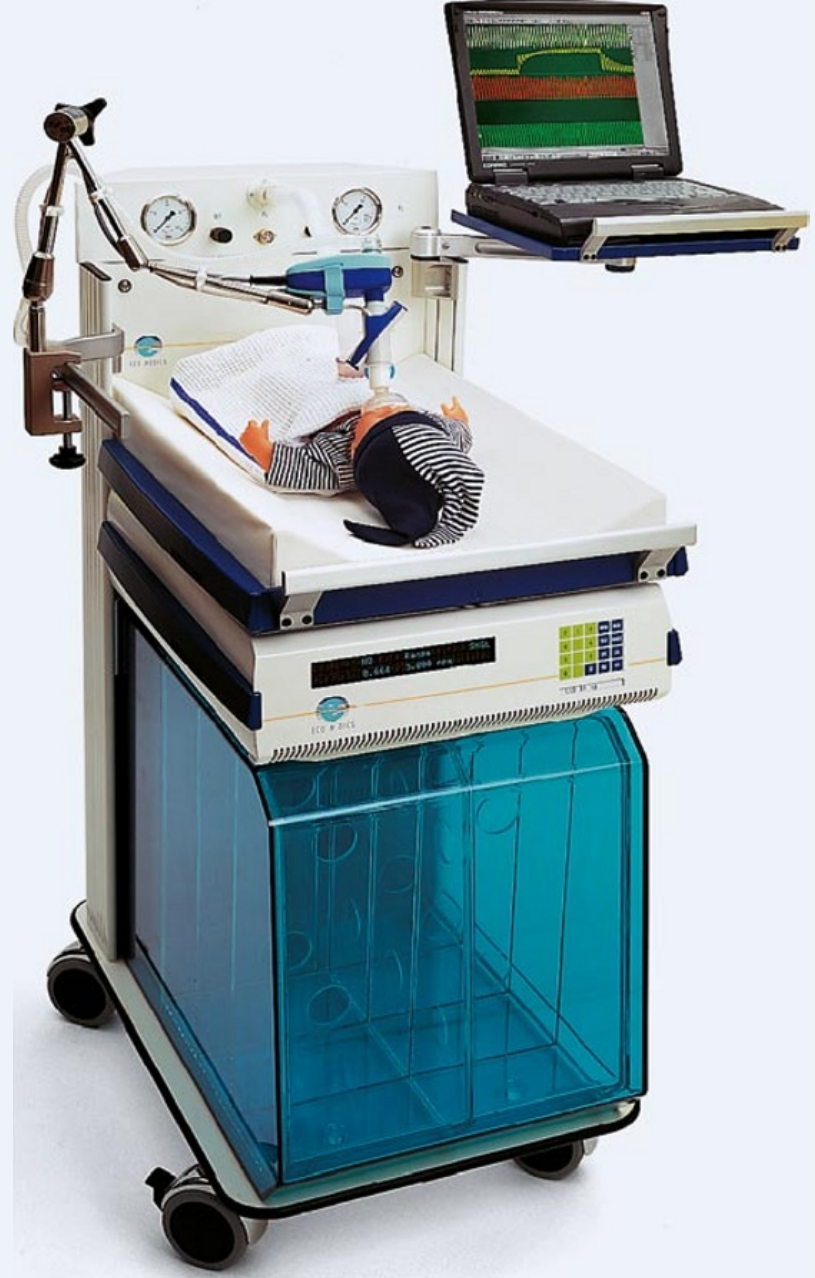

Abb. $2 \Delta$ Gerät zur Durchführung von „Multiple-breath-washout-Messungen". Mit dieser Methode kann sowohl das Lungenvolumen (FRC) als auch die Gasverteilung $(L C I)$ bestimmt werden lichste Parameter der Ventilationshomogenität ist der sog. „Lung-clearance-Index" (LCI), der gleichbedeutend ist mit der Anzahl von Atemzügen, die benötigt werden, um die FRC komplett aus der Lunge auszuwaschen. Während schon länger bekannt ist, dass der LCI bei älteren Kindern mit Mukoviszidose (CF) deutlich sensitiver den Zustand der Erkrankung anzeigt als herkömmliche Lungenfunktionsparameter, wie z. B. $\mathrm{FEV}_{1}$ [21], gibt es neuere Ergebnisse, die darauf hinweisen, dass die Gasverteilung bei CF-Patienten schon im Säuglingsalter gestört ist [23]. Auch bei Frühgeborenen mit CLD scheint die Inhomogenität der Gasverteilung ein wichtiger Marker für die Lungenentwicklung zu sein und ist möglicherweise von Nutzen für die Progno- se des Krankheitsverlaufes [25]. Neue, alternative Analysemethoden der einzelnen Atemzüge (sog. „slope-III-analysis“) lassen möglicherweise zusätzliche Aussagen über den Ort der gestörten Gasverteilung zu [14]. Noch ungeklärt bei sämtlichen Methoden der Gasverteilung ist dabei der genaue Einfluss des Testgases und der anteiligen Totraumventilation auf die Testergebnisse.

\section{Mechanik des respiratorischen Systems}

Die mechanischen Eigenschaften des respiratorischen Systems (Lunge, Luftwege und Thoraxwand) können durch dynamische oder statische Methoden gemessen werden, wobei die Techniken auf der
Messung von Atemfluss, Volumen und Druck basieren [12].

Folgende Techniken können im Säuglingsalter angewendet werden: Okklusionstechnik, Interruptionstechnik, Ganzkörperplethysmographie, Oszillationstechnik („forced oscillation technique“) sowie Thoraxkompressionstechnik. Alle Techniken beruhen auf Messungen von Fluss, Volumen und Druck. Bei der Okklusionstechnik und Interruptionstechnik kommt es während des Atemwegsverschlusses zu einem Druckausgleich zwischen Atemwegsöffnung und Alveolen. Der an der Atemwegsöffnung gemessene Druck $\left(\mathrm{p}_{\mathrm{ao}}\right)$ entspricht somit den elastischen Rückstellkräften des gesamten respiratorischen Systems („driving pressure"). 
Okklusionstechnik. Bei der „single occlusion technique" (SOT) wird mit einem kurzen endinspiratorischen Atemwegsverschluss der Hering-Breuer-Reflex (HBR) ausgelöst, der messbare Druck spiegelt somit die passive Mechanik während totaler Relaxation der Atemmuskulatur wieder [11]. Bei der „multiple occlusion technique" (MOT) werden mindestens 10 kurze Okklusionen an verschiedenen Volumenpunkten während der Exspiration durchgeführt. Das Volumen sowie der $\mathrm{p}_{\mathrm{a}}$ werden während des Atemwegsverschlusses gemessen und als Volumen-Druck-Paare gegeneinander aufgetragen, wobei die Steigung der resultierenden Gerade der Compliance des respiratorischen Systems entspricht [11].

Interruptionstechnik. Bei der Interruptionstechnik wird die „airway resistance “ $\left(\mathrm{R}_{\text {int }}\right)$ durch Atemwegsverschlüsse während der Exspiration der Ruheatmung bestimmt, ohne den HBR zu induzieren [17].

Ganzkörperplethysmographie. Das Prinzip der Atemwegswiderstandsmessung mittels Ganzkörperplethysmographie ist wie bei kooperativen Kindern: Während ruhiger Spontanatmung werden Druckänderungen im Plethysmographen und Flussänderungen aufgezeichnet und zu Druckänderungen an der Atemwegsöffnung während einer Atemwegsokklusion und den resultierenden Druckänderungen im Plethysmographen in Beziehung gesetzt.

Oszillationstechnik. Die Idee der Oszillationstechniken („,forced oscillation technique“) beruht darauf, dass die Lunge mit einem definierten Signal (z. B. Sinusschwingungen) angeregt wird und ihre Reaktion als mechanisches Verhalten interpretiert werden kann. Diese sog. Impedanz des respiratorischen Systems kann entweder als Inputimpedanz (Lautsprecheranregung und resultierende oszillatorische Flussschwingungen werden am Mund gemessen) oder als Transferimpedanz (Anregung per Lautsprecher an der Thoraxwand, Messung der resultierenden Flussschwingungen am Mund) bestimmt werden [8].

Thoraxkompressionstechnik. Bei der Thoraxkompressionstechnik („rapid tho-

Pneumologe 2007· 4:233-240 DOI 10.1007/s10405-007-0155-y

C) Springer Medizin Verlag 2007

P. Latzin · G. Hafen

Lungenfunktion im Säuglings- und Kleinkindalter

\section{Zusammenfassung}

Wachstum und Entwicklung der Atemwege während der perinatalen Phase sind wegweisend für die respiratorische Morbidität des gesamten Lebens. Lungenfunktionsuntersuchungen können bereits zu solch frühen Zeitpunkten Aufschluss über die Funktion der Atemwege und damit neben der klinischen Beurteilung in unklaren Situationen zusätzliche Hinweise auf mögliche Erkrankungen geben. Die Basisuntersuchung stellt die FlussVolumen-Kurve während der Ruheatmung dar. Als weiteres Standardverfahren ist die Volumenmessung etabliert. Wenn hierbei die Gasverdünnungsmethode gewählt wird, können zusätzlich Aussagen über die Venti-

\section{Pulmonary function in babies and infants}

\section{Abstract}

The growth and development of the airways during the perinatal period have lifelong consequences for respiratory morbidity. In unclear situations, measurements of infant pulmonary function can, in addition to clinical judgment, provide useful information about possible disease mechanisms even at this early stage. The flow-volume loop is the most widely used test; another well standardised method is the measurement of pulmonary volume. When assessed by the gas dilution method, additional information on gas mixing and ventilation inhomogeneity can be lationsinhomogenität der Lunge gewonnen werden. Messungen der NO-Konzentration in der Ausatemluft gewinnen zunehmend an Bedeutung, da sie wichtige Information über Atemwegsentzündungen liefern. In diesem Beitrag werden die gängigen Methoden der Lungenfunktionsmessung im Säuglingsalter dargestellt, ihre Anwendungsbereiche erläutert und aktuelle Entwicklungen und Ergebnisse präsentiert.

Schlüsselwörter

Lungenfunktion · Kind · Fluss-Volumen-

Kurve · Lungenvolumen $\cdot$ NO obtained. Measurement of nitric oxide in exhaled air is becoming increasingly important, as airway inflammation can be determined easily and very reliably by this method. This review describes these and other commonly used methods for testing infant pulmonary function, discusses their use in practice, and provides information on current developments and the latest findings.

\section{Keywords} Pulmonary function · Child · Flow-volume loop Lung volume $\cdot$ Nitric oxide 


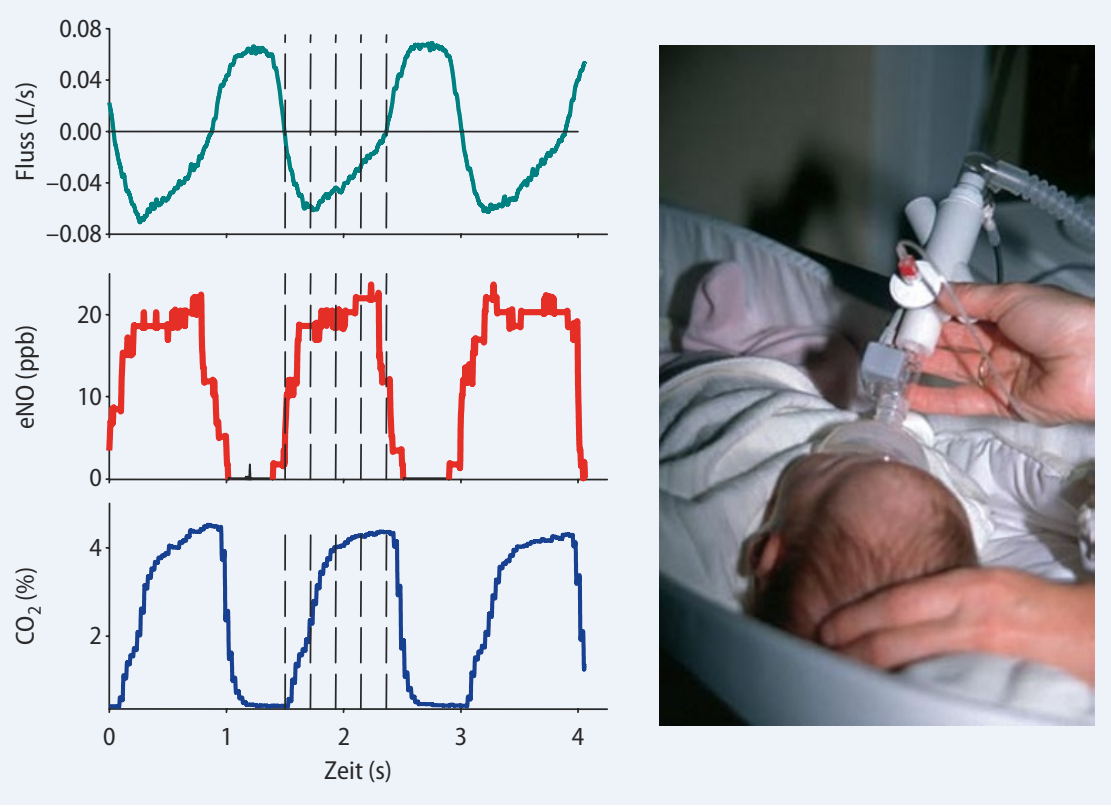

Abb. $3 \Delta$ Durchführung der NO-Messung bei einem 4 Wochen alten Säugling. Fluss-Volumen-Kurve und $\mathrm{CO}_{2}$ werden im Hauptstrom gemessen, parallel dazu wird über einen zusätzlichen Abnehmer (im Bild roter Filter) die NO-Konzentration bestimmt. Damit kann der sog. NO-Output berechnet werden

racic compression technique", RTC) wird die Spirometrie des kooperativen Kindes mit der Möglichkeit einer forcierten exspiratorischen Fluss-Volumen-Kurve imitiert. Dabei wird beim sedierten Säugling mittels einer aufblasbaren Weste ein externer Druck auf Thorax und Abdomen ausgeübt, um eine forcierte Exspiration am Ende einer Inspiration zu produzieren. Heute wird oft die „raised volume RTC“ verwendet, da die „volle“ forcierte Fluss-Volumen-Kurve weniger volumenabhängig ist.

\section{Entzündung}

Das Ausmaß einer möglichen Atemwegsentzündung bestimmen zu können ist insbesondere wichtig, um zugrunde liegende Mechanismen verschiedener Atemwegserkrankungen, wie z. B. Asthma bronchiale, zu erfassen. Nachdem Messungen von Stickstoffmonoxid in der Ausatemluft (NO) bei Erwachsenen und älteren Kindern mittlerweile Standard bei Diagnostik und Therapiekontrolle von Asthmatikern sind [1], gab es erst in den letzten Jahren Möglichkeiten, auch bei Säuglingen NO standardisiert und unter Berücksichtigung des exspiratorischen Flusses zu bestimmen [16] Mehrere Gruppen nutzen diese Technik heutzutage: Dabei wird der mittle- re NO-Wert während des 3. Quartals des Ausatemzyklus berechnet. Angepasst an den exspiratorischen Fluss kann so auch der sog. NO-Output bestimmt werden (• Abb. 3). Unter anderem konnte mithilfe dieser Methode gezeigt werden, dass erhöhte NO-Werte nach Geburt bei Kindern von atopischen Müttern in Zusammenhang mit vermehrten Atemwegssymptomen während des 1 . Lebensjahres stehen [22]. Auch wenn bei dieser "Multiple-breath-Methode“ nicht exakt zwischen oraler und nasaler NO-Herkunft unterschieden werden kann, könnte die Bedeutung von NO-Messungen im Kleinkindalter gerade in der Diagnostik bzw. dem Ausschluss von Zilienfunktionsstörungen liegen [28].

\section{$\checkmark$ NO-Messungen im Kleinkindalter könnten Bedeutung in der Diagnostik von Zilienfunktionsstö- rungen erlangen}

Bei Erwachsenen und älteren Kindern hat die Sammlung von Atemkondensat zur Bestimmung von Entzündungsparametern in den letzten 10 Jahren zunehmend an Bedeutung gewonnen, auch wenn dabei fehlende Standardisierung und viele offene Fragen die Genauigkeit der Resultate noch unklar erscheinen lassen [18].
Trotz einiger Versuche, diese Methode auch im Kleinkindalter zu etablieren [13] erscheinen uns oben erwähnte Probleme momentan noch zu groß, um zuverlässige Ergebnisse zu erzielen. Eventuell kann die sog. „electronic nose“ dieser eigentlich vielversprechenden nichtinvasiven Bestimmung von Entzündungsparametern einen weiteren Entwicklungsschub geben [30].

\section{Neue Methoden}

Der Trend zu noch weniger invasiven und praktischen Messungen setzt sich auch bei der Lungenfunktionsdiagnostik im Säuglingsalter fort. Dabei wurden in den letzten Jahren v. a. 2 Methoden für den Einsatz im Säuglingsalter (weiter)entwickelt, die sog. ,electrical-impedance-tomography" (EIT) und das Elektromyogramm (EMG). Mit der bildgebenden Darstellung des EIT können Hinweise auf Zusammenhänge zwischen Struktur und Funktion erlangt werden. Der Nachteil beider Methoden liegt darin, dass große individuelle Unterschiede in der Grundaktivität vorliegen, sodass sie momentan eher für intraindividuelle Beobachtungen von Interesse sind (• Tab. 2).

\section{Fazit für die Praxis}

Die Lungenfunktionsprüfung im Säuglings- und Kleinkindalter ist im letzten Jahrzehnt den Kinderschuhen entwachsen. Standardisierte Messbedingungen und kommerziell erhältliche Geräte haben ebenso wie neue, weniger invasive Messmethoden dazu beigetragen. Obwohl die Bedeutung des respiratorischen Zustandes früh nach Geburt für das weitere Leben immer deutlicher wird, bleiben Lungenfunktionsprüfungen im Säuglings- und Kleinkindalter durch den relativ hohen zeitlichen und finanziellen Aufwand vorerst spezialisierten Zentren vorbehalten. Bei weiterer Vereinfachung der NO-Messungen könnte sich dies jedoch bald ändern, v. a. weil die einfache und relativ genaue Diagnostik von Atemwegsentzündungen und Zilienfunktionsstörungen im Säuglingsalter eine direkte Konsequenz für den behandelnden Kinderpneumologen hätte. 
Hier steht eine Anzeige.

黛 Springer 


\section{Korrespondenzadresse Dr. P. Latzin}

Abteilung für pädiatrische
Pneumologie, Inselspital,
Universitätskinderklinik Bern
3010 Bern
Schweiz
philipp.latzin@insel.ch

Danksagung. Die Autoren bedanken sich bei Prof. Dr. Urs Frey für die Unterstützung beim Erstellen des Artikels.

Interessenkonflikt. Es besteht kein Interessenkonflikt. Der korrespondierende Autor versichert, dass keine Verbindungen mit einer Firma, deren Produkt in dem Artikel genannt ist, oder einer Firma, die ein Konkurrenzprodukt vertreibt, bestehen. Die Präsentation des Themas ist unabhängig und die Darstellung der Inhalte produktneutral.

\section{Literatur}

1. ATS/ERS Recommendations for Standardized Procedures for the Online and Offline Measurement of Exhaled Lower Respiratory Nitric Oxide and Nasal Nitric Oxide, 2005. Am J Respir Crit Care Med 171: 912-930

2. Aurora P, Kozlowska W, Stocks J (2005) Gas mixing efficiency from birth to adulthood measured by multiple-breath washout. Respir Physiol Neurobio 148: 125-139

3. Bates JH, Schmalisch G, Filbrun D, Stocks J (2000) Tidal breath analysis for infant pulmonary function testing. ERS/ATS Task Force on Standards for Infant Respiratory Function Testing. European Respiratory Society/American Thoracic Society. Eur Respir J 16: $1180-1192$

4. Buess C, Pietsch P, Guggenbuhl W, Koller EA (1986) A pulsed diagonal-beam ultrasonic airflow meter. J Appl Physiol 61: 1195-1199

5. Dezateux C, Stocks J (1997) Lung development and early origins of childhood respiratory illness. Br Med Bull 53: 40-57

6. Dezateux C, Stocks J, Wade AM et al. (2001) Airway function at one year: association with premorbid airway function, wheezing, and maternal smoking. Thorax 56: 680-686

7. Filippone M, Sartor M, Zacchello F, Baraldi E (2003) Flow limitation in infants with bronchopulmonary dysplasia and respiratory function at school age. Lancet 361: 753-754

8. Frey U (2005) Forced oscillation technique in infants and young children. Paediatr Respir Rev 6: 246-254

9. Frey U, Stocks J, Coates A et al. (2000) Specifications for equipment used for infant pulmonary function testing. ERS/ATS Task Force on Standards for Infant Respiratory Function Testing. European Respiratory Society/American Thoracic Society. Eur Respir J 16: 731-740

10. Frey U, Stocks J, Sly P, Bates J (2000) Specification for signal processing and data handling used for infant pulmonary function testing. ERS/ATS Task Force on Standards for Infant Respiratory Function Testing. European Respiratory Society/American Thoracic Society. Eur Respir J 16: 1016-1022

11. Gappa M, Colin AA, Goetz I, Stocks J (2001) Passive respiratory mechanics: the occlusion techniques. Eur Respir J 17: 141-148
12. Gappa M, Pillow JJ, Allen J et al. (2006) Lung function tests in neonates and infants with chronic lung disease: lung and chest-wall mechanics. Pediatr Pulmonol 41: 291-317

13. Griese M, Latzin P, Beck J (2001) A noninvasive method to collect nasally exhaled air condensate in humans of all ages. Eur J Clin Invest 31: 915-920

14. Gustafsson PM (2007) Peripheral airway involvement in CF and asthma compared by inert gas washout. Pediatr.Pulmonol 42: 168-176

15. Haland G, Carlsen KC, Sandvik L et al. (2006) Reduced lung function at birth and the risk of asthma at 10 years of age. $N$ Engl J Med 355: 1682-1689

16. Hall GL, Reinmann B, Wildhaber JH, Frey U (2002) Tidal exhaled nitric oxide in healthy, unsedated newborn infants with prenatal tobacco exposure. J Appl Physiol 92: 59-66

17. Hall GL, Wildhaber JH, Cernelc M, Frey U (2001) Evaluation of the interrupter technique in healthy, unsedated infants. Eur Respir J 18: 982-988

18. Horvath I, Hunt J, Barnes PJ (2005) Exhaled breath condensate: methodological recommendations and unresolved questions. Eur Respir J 26: 523548

19. Hulskamp G, Hoo AF, Ljungberg H et al. (2003) Progressive decline in plethysmographic lung volumes in infants: physiology or technology? Am J Respir Crit Care Med 168: 1003-1009

20. Hulskamp G, Pillow JJ, Dinger J, Stocks J (2006) Lung function tests in neonates and infants with chronic lung disease of infancy: functional residual capacity. Pediatr Pulmonol 41: 1-22

21. Kraemer R, Blum A, Schibler A et al. (2005) Ventilation inhomogeneities in relation to standard lung function in patients with cystic fibrosis. Am J Respir Crit Care Med 171: 371-378

22. Latzin P, Kuehni CE, Baldwin DN et al. (2006) Elevated exhaled nitric oxide in newborns of atopic mothers precedes respiratory symptoms. Am J Respir Crit Care Med 174: 1292-1298

23. Lum S, Gustafsson P, Ljungberg H et al. (2007) Early detection of cystic fibrosis lung disease: multiplebreath washout vs. raised volume tests. Thorax 62 : 341-347

24. Morris MG, Gustafsson P, Tepper R et al. (2001) The bias flow nitrogen washout technique for measuring the functional residual capacity in infants. ERS/ATS Task Force on Standards for Infant Respiratory Function Testing. Eur Respir J 17: 529-536

25. Pillow JJ, Frerichs I, Stocks J (2006) Lung function tests in neonates and infants with chronic lung disease: global and regional ventilation inhomogeneity. Pediatr Pulmonol 41: 105-121

26. Rieger C, Hardt H. v.d., Sennhauser FH et al. (2004) Pädiatrische Pneumologie, 2. Aufl. Springer, Berlin Heidelberg New York Tokyo

27. Schibler A, Hall GL, Businger F et al. (2002) Measurement of lung volume and ventilation distribution with an ultrasonic flow meter in healthy infants. Eur Respir J 20: 912-918

28. Stehling F, Roll C, Ratjen F, Grasemann H (2006) Nasal nitric oxide to diagnose primary ciliary dyskinesia in newborns. Arch Dis Child Fetal Neonatal Ed 91: F233

29. Stocks J, Godfrey S, Beardsmore C et al. (2001) Plethysmographic measurements of lung volume and airway resistance. ERS/ATS Task Force on Standards for Infant Respiratory Function Testing. European Respiratory Society/ American Thoracic Society. Eur Respir J 17: 302-312

30. Thaler ER, Hanson CW (2005) Medical applications of electronic nose technology. Expert Rev Med Devices 2: 559-566

\section{Aktuelle Informationen zum Thema Tuberkulose veröffentlicht}

Das Deutsche Zentralkomitee zur Bekämpfung der Tuberkulose (DZK) hat seinen 30 . Informationsbericht herausgegeben.

Der Bericht enthält auf 85 Seiten aktuelle Beiträge zu Problemen in der weltweiten Bekämpfung der Tuberkulose sowie Eckdaten zur Tuberkulose in Deutschland für das Jahr 2005. Weiterhin bietet er eine Übersicht zum aktuellen Stand und den internationalen Empfehlungen zu Interferon-Gamma-Tests zur Diagnose der tuberkulösen Infektion. Ein weiterer Beitrag beschäftigt sich mit der Diagnose der latenten Tuberkulose-Infektion bei Beschäftigten im Gesundheitswesen. Zudem enthält der Bericht einen Kommentar zu den neuen Empfehlungen für die Umgebungsuntersuchungen bei Tuberkulose und einen Beitrag zur Tuberkulose in deutschen und europäischen Gefängnissen. Eine weitere Übersicht veranschaulicht die Ergebnisse der DZK-Studie zur molekularen Epidemiologie der Tuberkulose in Deutschland.

Der DZK-Bericht stellt für den TuberkuloseInteressierten eine informative Lektüre zu wichtigen aktuellen Fragen dar. Er kann zum Preis von EUR 15,00 zzgl. Porto beim DZK bestellt werden:

Deutsches Zentralkomitee zur Bekämpfung der Tuberkulose

Lungenklinik Heckeshorn

HELIOS Klinikum Emil von Behring

Walterhöferstr. 11

14165 Berlin

Tel.: (030) 8102-1164

Fax: (030) 8102-1362

E-Mail: rloddenkemper@dzk-tuberkulose.de

Quelle: Deutsches Zentralkomitee zur Bekämpfung der Tuberkulose, www.dzk-tuberkulose.de 